\title{
ATIVIDADE DE FUNGOS NEMATÓFAGOS NOS ESTÁGIOS PRÉ- PARASITÁRIOS DE NEMATÓDEOS TRICHOSTRONGILÍDEOS
}

\author{
PREDACIOUS ACTIVITY OF NEMATOPHAGOUS FUNGI \\ ON FREE LIVING STAGES OF TRICHOSTRONGYLID NEMATODES
}

\author{
Terezinha Padilha ${ }^{1}$
}

- REVISÃo BIBLIOGRÁFICA -

\section{RESUMO}

$O$ controle dos nematódeos trichostrongilideos é obtido através da aplicação de anti-helminticos nos hospedeiros. Nas últimas décadas, estratégias de controle foram desenvolvidas visando a aplicação de anti-helmínticos em épocas em que as condições ambientais são desfavoráveis ao desenvolvimento dos estádios pré-parasitários. Estas estratégias apesar de serem bem sucedidas, apresentam inconveniências tais como o desenvolvimento de resistência aos principios ativos mais comumente usados, a possibilidade de ocorrerem residuos na carne e no leite e a ecotoxicidade de alguns compostos. Esses problemas reais e potenciais tem impulsionado estudos visando o desenvolvimento de alternativas que possam contribuir para redução ou substituição do uso desses compostos. Entre elas, o controle biológico dos estádios pré-parasitários através da ação de fungos nematófagos é uma alternativa promissora. Este artigo reúne as principais informações geradas nos estudos com estes microrganismos visando a redução do número de larvas de nematódeos trichostrongilideos disponiveis nas pastagens.

Palavras-chave: Nematódeos trichostrongilideos, controle biológico, fungos nematófagos.

\section{SUMMARY}

Nematophagous fungi are promising candidates for use as biological control agents to reduce the numbers of infective larvae of trichostrongylid on pasture. In the last five

\begin{abstract}
years research aimed at the use of nematophagous fungi to control trichostrongylid nematodes has been intensified. This paper presents a review of the current status of knowledge on the effect of this group of microorganism on the free living stages of these nematodes.
\end{abstract}

Key words: Trichostrongylid nematodes, biological control, nematophagous fungi

\section{INTRODUÇÃO}

A redução do número de larvas infectantes de nematódeos nas pastagens é um dos objetivos do controle das verminoses. A redução da contaminação das pastagens é obtida através da aplicação de antihelmínticos nos hospedeiros nas épocas em que as condições ambientais são desfavoráveis ao desenvolvimento dos estádios de vida livre. Essas aplicações visam a eliminação da grande maioria da população de adultos no hospedeiro. Assim, uma menor quantidade de ovos é passada ao exterior, onde as condições de desenvolvimento e sobrevivência são prejudiciais aos estádios préparasitários, ocorrendo portanto uma redução da população de larvas disponíveis.

\footnotetext{
Médico Veterinário, PhD, Pesquisadora da EMBRAPA - Centro Nacional de Pesquisa de Gado de Leite, Rodovia MG 133 km 42, Coronel Pacheco MG 36.155-000, Bolsista do CNPq
} 
A estratégia de redução das larvas infectantes nas pastagens utilizada nos esquemas de controle estratégicos é bem sucedida. Entretanto, o aparecimento de resistência aos princípios ativos anti-helmínticos, a existência de resíduos na carne e no leite e a ecotoxicidade de alguns compostos despertaram o interesse no desenvolvimento de alternativas que possam contribuir para minimizar o uso de anti-helmínticos no controle das verminoses (HERD et al., 1993; BJØRN, 1994; DONALD, 1994; PRICHARD, 1994; WALLER, 1994).

Durante o desenvolvimento no meio ambiente, os estádios pré-parasitários dos nematódeos trichostrongilídeos são submetidos ao efeito de fatores abióticos, como temperatura, umidade, tensão de oxigênio, assim como fatores bióticos como ácaros, bactérias, fungos, vírus e outros agentes. Para que haja continuidade do ciclo biológico, os estádios de vida livre necessitam superar as barreiras causadas por esses fatores. A busca de alternativas para o controle da verminose dos ruminantes aponta para a necessidade de identificação de mecanismos que promovam o aumento ou a potencialização de fatores adversos ao ciclo biológico, como estratégia importante para a redução da contaminação das pastagens e conseqüentemente da população de helmintos nos animais em pastoreio.

A possibilidade de utilização do controle biológico na profilaxia das verminoses é uma alternativa promissora. Para isto, agentes biológicos com ação sobre ovos e larvas seriam aplicados nas pastagens ou administrados aos animais em épocas estratégicas a serem definidas de acordo com a epidemiologia das verminoses e a biologia dos agentes. Eles exerceriam sua ação sobre os ovo: e larvas, promovendo a redução da contaminação através de mecanismos capazes de determinai a mortalidade dos ovos ou larvas ou interferir em funções vitais que ocasionem alterações no comportamento laryar.

A utilização de agentes biológicos com ação nos ovos e larvas de nematódeos trichostrongilídeos como alternativa para higienização das pastagens tem sido estudada em alguns laboratórios. Os fungos nematófagos são os microrganismos mais estudados com este objetivo. Esses fungos vivem na matéria orgânica do solo onde desenvolveram relações parasíticas ou predatórias com os nematódeos (BARRON, 1977). Este artigo reúne as principais informações disponíveis sobre estes microrganismos com o propósito de reduzir o número de larvas de nematódeos trichostrongilídeos disponíveis nas pastagens.

\section{FUNGOS OVICIDAS}

Os fungos nematófagos ovicidas produzem hifas que fixam-se nos ovos (Figura 1). $O$ ataque de fungos em ovos de Ascaris lumbricoides foi estudado através da microscopia eletrônica (LÝSEK \& KRAJŠÍ, 1987). Inicialmente, é estabelecido um ponto de contato entre a hifa e a superfície do ovo. Em seguida, o fungo forma uma dilatação nesse ponto e danifica o complexo quitina-proteína da casca do ovo, provavelmente mediante a ação de enzimas, facilitando a penetração. Após a penetração, o fungo forma ramos micelares no interior do ovo consumindo o embrião, que pode sofrer $o$ ataque em qualquer estágio de desenvolvimento. Nenhuma informação sobre a ação desses fungos em nematódeos trichostrongilídeos é ainda disponível. É importante notar que os ovos de trichostrongilídeos se desenvolvem e eclodem em cerca de 12-24 horas após a deposição dos bolos fecais nas pastagens. Logo, os fungos ovicidas provavelmente não tenham tempo de atuar sobre os ovos desses nematódeos.

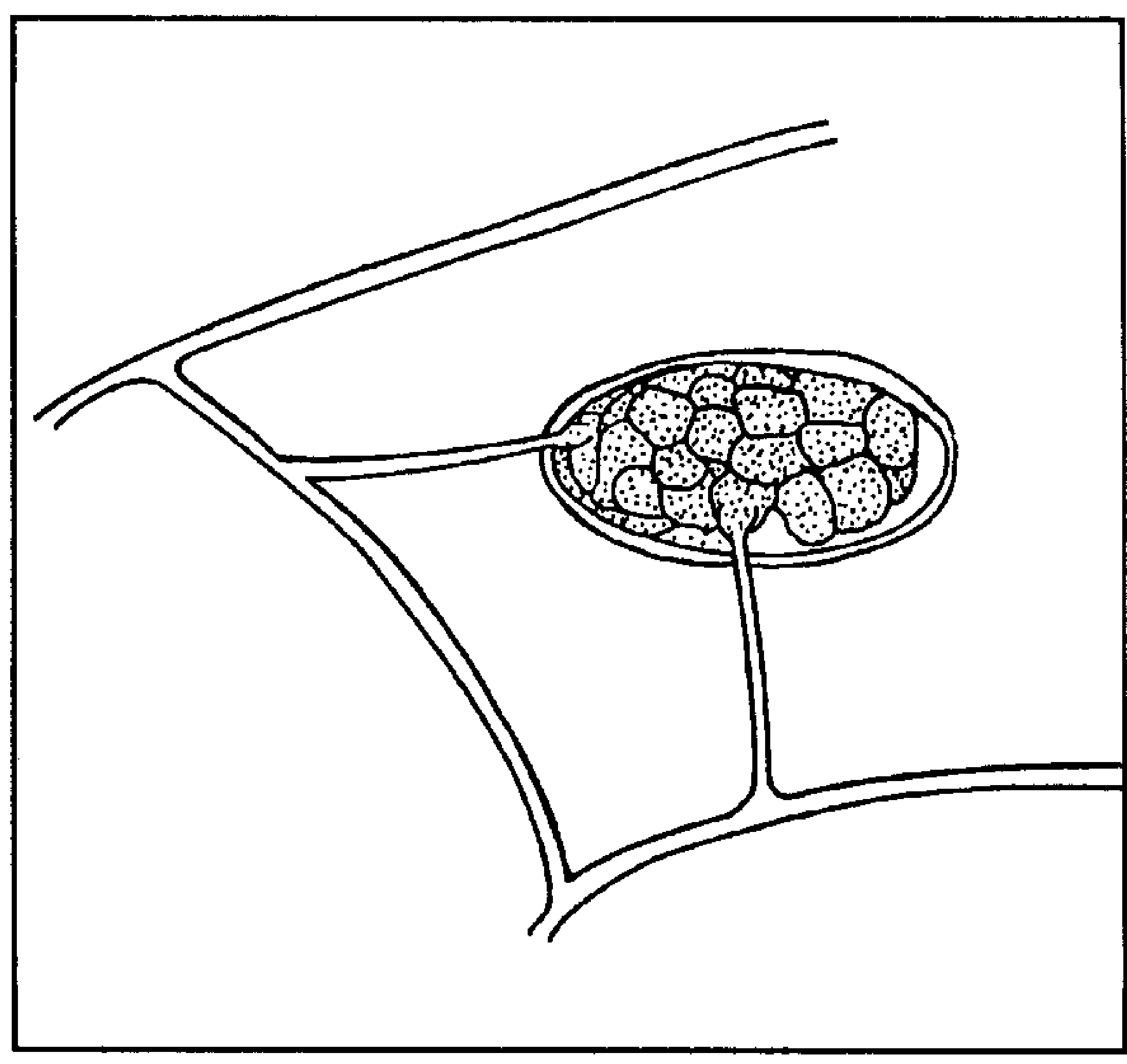

Figura 1. Infecção de ovos de nematódeos por hifas de fungos ovicidas (adaptado de BARRON, 1977 com permissão).

\section{FUNGOS ENDOPARASITAS}

Os fungos endoparasitas desenvolvem-se no interior do corpo do nematódeo, sendo o esporo a forma infectante. A infecção inicia-se com a adesão do esporo à cutícula do nematódeo (esporo 
adesivo) ou através da ingestão. Em seguida, o esporo germina, penetra na cavidade do corpo onde produz o talo infeccioso que cresce e absorve $o$ conteúdo corporal do nematódeo (BARRON, 1977; DIJKSTERHUIS et al., 1991). Os efeitos dos fungos endoparasitas nas formas de vida livre de nematódeos trichostrongilídeos não são extensivamente conhecidos. JANSSON et al. (1985) tentaram infectar, sem sucesso, larvas de Ostertagia ostertagi, Ostertagia circumcincta, Haemonchus contortus e Trichostrongylus colubriformis com Drechmeria coniospo$r a$. Contudo, quando a cutícula da larva de segundo estádio foi removida, o fungo foi capaz de infectar. Nas larvas de primeiro estádio de $\boldsymbol{H}$. contortus, os conídios de $\boldsymbol{D}$. coniospora aderem-se a região cefálica, nas proximidades da cápsula bucal (SANTOS \& CHARLES, 1995a). Resultados dos testes sobre o efeito desse endoparasita no desenvolvimento de estádios de vida livre de $\boldsymbol{H}$. contortus mostraram que ele é eficiente quando encontra-se em altas concentrações provavelmente devido a necessidade de haver um maior contato físico entre o nematódeo e o fungo, para que ocorra a infecção. Logo, quanto maior o número de conídios maior a chance de ocorrer a infecção (SANTOS \& CHARLES, 1995a). Em um teste onde $10^{5}, 10^{6}$ e $10^{8}$ conídios foram adicionados em cada grama de fezes de ovinos contendo ovos de $\boldsymbol{H}$. contortus, apenas a concentração de $10^{8}$ conídios por grama apresentou redução significativa $(88,4 \%)$ (SANTOS \& CHARLES, 1995a).

$\mathrm{O}$ efeito de um outro fungo endoparasita foi recentemente estudado. No estudo, conídios de Harposporium anguillulae, um endoparasita que coloniza rapidamente os bolos fecais de bovinos depositados em pastagens de Brachiaria decumbens na Zona da Mata de Minas Gerais, foram adicionados a fezes ovinas contendo ovos de $\boldsymbol{H}$. contortus. Nos cultivos que receberam conídios de $\boldsymbol{H}$. anguillulae, o número médio de larvas recuperadas foi de três larvas em cada grama de fezes, enquanto nos cultivos não tratados o número médio de larvas encontrado foi de 594, representando uma redução de $99,5 \%$ (CHARLES et al., 1995).

Os fungos endoparasitas são eficientes e algumas espécies produzem grande quantidade de conídios e clamidosporos (BARRON, 1977), características importantes para que uma espécie possa ser utilizada no controle biológico. Entretanto, eles são parasitas obrigatórios, o que poderá limitar a sua utilização, já que grande quantidade de inócuo precisa ser produzida para uso em grande escala.

\section{FUNGOS PREDADORES}

\section{Mecanismo de ação}

Os fungos predadores produzem grande quantidade de hifas que se modificam em estruturas especializadas (armadilhas) com a finalidade de capturar e fixar nematódeos (BARRON, 1977). Essas estruturas podem ser aderentes ou não e serem produzidas espontaneamente ou em resposta a presença de nematódeos. Vários tipos de armadilhas podem ser desenvolvidos pelos fungos (Figuras $2 \mathrm{e}$ 3 ). Nos que utilizam armadilhas aderentes, a substância adesiva pode cobrir toda a hifa ou apenas as estruturas especializadas e/ou as redes. As estruturas de captura não aderentes são os anéis constritores e os não constritores. Os anéis constritores tem ação ativa, geralmente têm três células e quando o nematódeo penetra no anel as células se expandem promovendo estrangulamento. Os anéis não constritores são estruturas passivas. Os nematódeos ao penetrarem nesses anéis, se enrolam e não conseguem sair.

A formação de armadilhas era atribuída apenas às hifas. Mais recentemente, DACKMAN \& NORDBRING-HERTZ (1992) provaram que os esporos também podem produzir armadilhas. Eles mostraram que conídios de Arthrobotrys oligospora germinam diretamente em armadilhas aderentes quando adicionados em placas de ágar nas proximidades de fezes bovinas. Estas armadilhas são produzidas, possivelmente em resposta a substâncias estimulantes que se difundem das fezes. Segundo os autores, a possibilidade de formação de armadilhas a partir dos conídios é uma característica que permite ao fungo sobreviver à ação fungicida natural existente no meio ambiente.

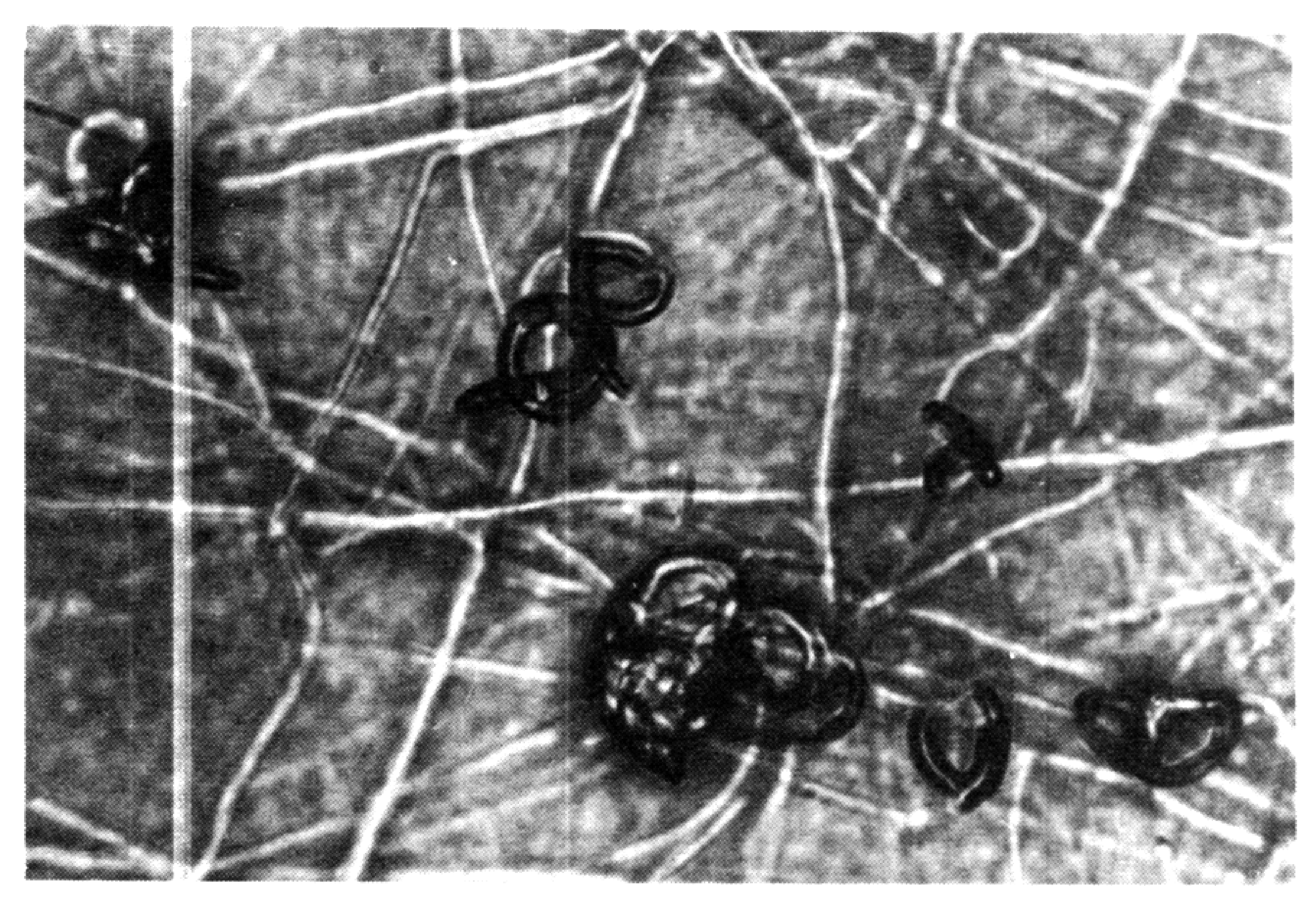

Figura 2. Superficie de ágar contendo cultivo de Arthrobotrys conoides, mostrando armadilhas em forma de redes tridimensionais utilizadas por esta espécie de fungo na captura de nematódeos (cedida pelo Dr. Pedro Mendoza-de-Gives, Morelos, México). 


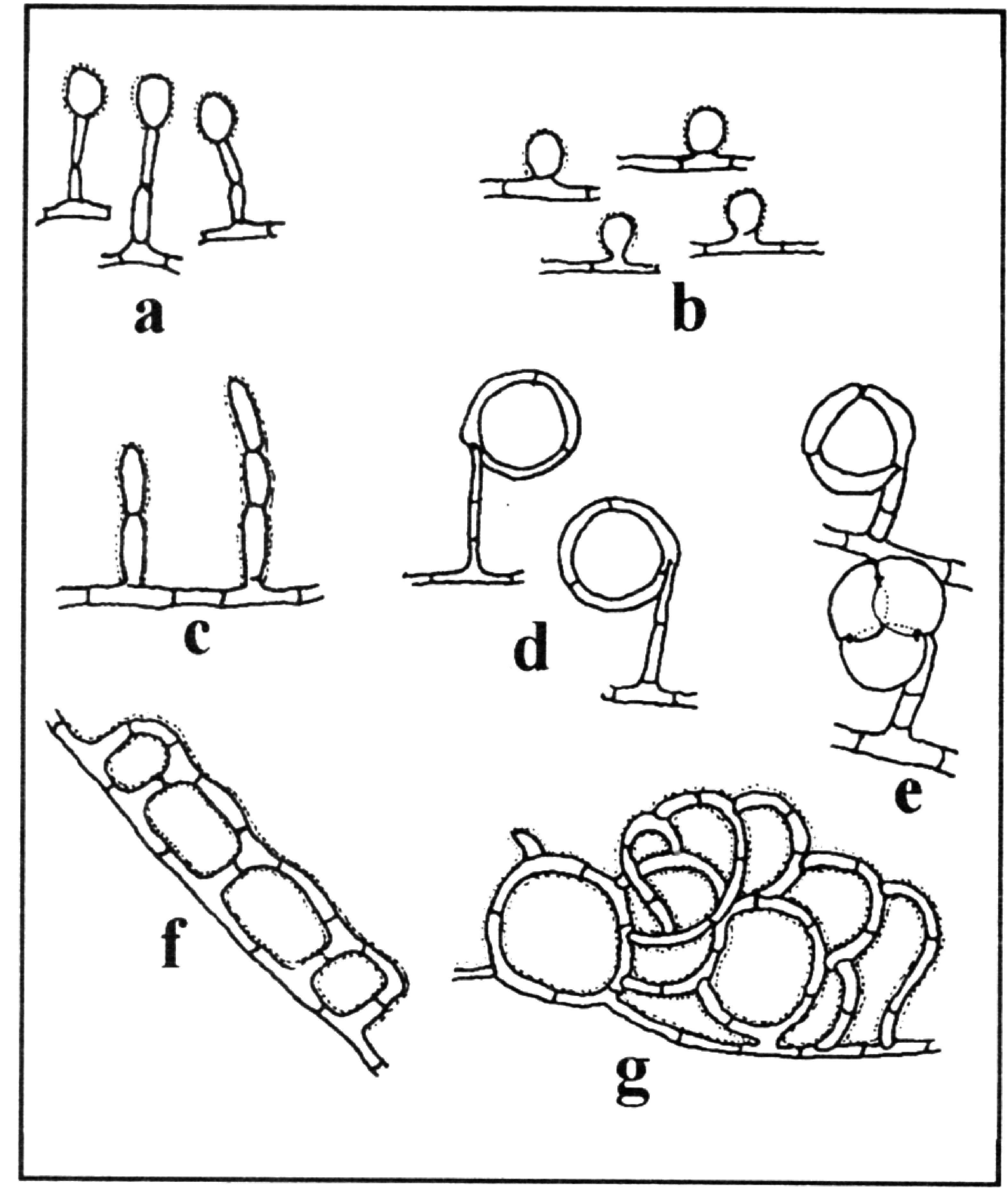

Figura 3. Tipos de armadilhas utilizadas por fungos nematófagos (adaptado de BARRON, 1977, com permissão): a) nódulos adesivos pedunculados, b) nódulos adesivos sésseis, c) hifas adesivas não modificadas, d) hifas modificadas em anéis não constritores, e) hifas modificadas em anéis constritores (abertos e fechados), f) hifas adesivas modificadas em redes bidimensionais, g) hifas adesivas modificadas em redes tridimensionais.

Ação de fungos do gênero Arthrobotrys em estágios pré-parasitários de trichostrongilídeos

Os fungos do gênero Arthrobotrys, capturam as larvas nas armadilhas aderentes ou não aderentes (Figura 4) e produzem substâncias hidrolíticas que auxiliam na imobilização e infecção. Estudos bioquímicos e ultra-estruturais mostraram que a adesão entre o fungo e o nematódeo inicia-se com o contato físico entre a superfície do nematódeo e a do fungo. Este contato induz a vários eventos como a interação entre os receptores de lecitina, a modificação de polímeros da superfície e a ativação de enzimas específicas. Como resultado, ocorre uma ligação firme entre o nematódeo e o fungo. Após a captura, o fungo penetra na cutícula da larva, formando um bulbo de infecção onde inicia-se o crescimento da hifa, culminando com o preenchimento de todo o corpo do nematódeo (NORDBRING-HERTZ, 1988; TUNLID \& JANSSON, 1991; TUNLID et al., 1992).

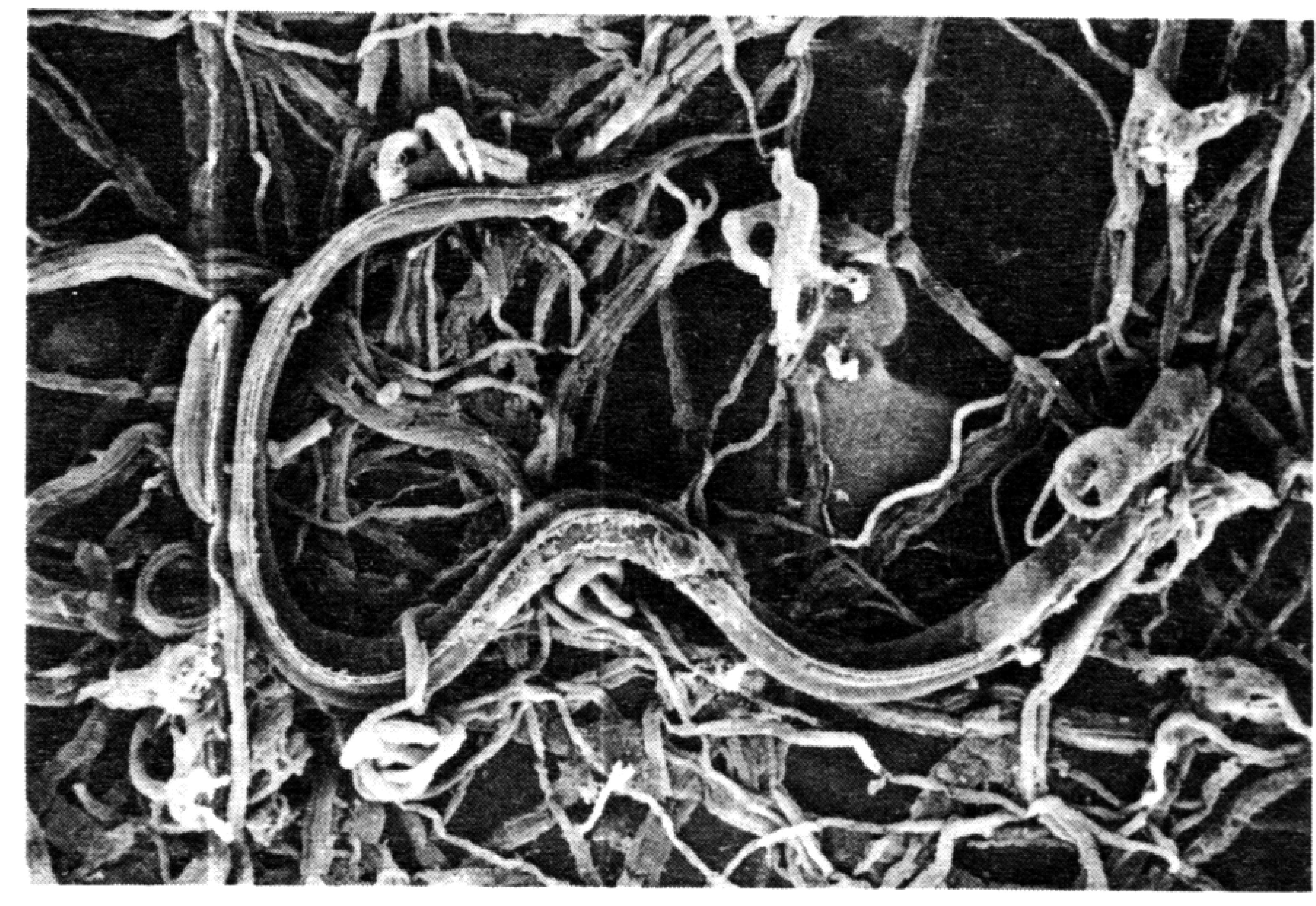

Figura 4. Larva infectante de Haemonchus contortus capturada pelo fungo Arthrobotrys conoides (cedida pelo Dr. pedro Mendoza-de-Gives, Morelos, México).

A ação do fungo $\boldsymbol{A}$. oligospora nas larvas de nematódeos trichostrongilídeos é a mais estudada. Uma série de artigos publicados por autores dinamarqueses caracterizaram a ação desse fungo tanto em cultivos de fezes quanto em bolos fecais mantidos no meio ambiente. A maioria das informações foi obtida usando uma cepa isolada na Suécia e larvas dos nematódeos Cooperia oncophora e $\boldsymbol{O}$. ostertagi. Primeiramente a capacidade do fungo em formar armadilhas na presença de larvas infectantes de nematódeos trichostrongilídeos foi observada em alguns experimentos usando-se o isolado sueco. NANSEN et al. (1986) demonstraram que larvas de primeiro e segundo estágio e larvas infectantes de $\boldsymbol{C}$. oncophora estimulam a formação de armadilhas que inicia-se entre três a seis horas após o contato com o fungo. Em cerca de 12-15 horas a quase totalidade das larvas foi presa nas armadilhas produzidas pelo fungo. Em seguida, NANSEN et al. (1988) testaram o fungo na presença de larvas infectantes de nove espécies de nematódeos e verificaram que as larvas de maior motilidade, como as de $\boldsymbol{C}$. oncophora, $\boldsymbol{O}$. ostertagi, Cooperia curticei e $\boldsymbol{H}$. contortus, incitam o fungo à formar armadilhas mais rapidamente que quando estimulados por larvas pouco ativas como as de Dictyocaulus viviparus.

Assim que a larva é aprisionada nas armadilhas, o fungo inicia o processo de infecção. Um bulbo infeccioso é formado, por onde crescem as hifas que preenchem o corpo do nematódeo. Quando o fungo infecta as larvas de primeiro ou segundo estágio elas morrem rapidamente. Entretanto, as larvas infectantes permanecem se movimentando nas armadilhas por cerca de 20 horas, provavelmente devido a dificuldade de penetração na cutícula da larva que é recoberta pela cutícula morta externa 
oriunda da larva de segundo estágio (NANSEN et al., 1986).

Redução do número de larvas infectantes em cultivos de fezes, bolos fecais e pastagens

A adição de conídios de $A$. oligospora reduz significativamente a população de larvas nas fezes. Para Cooperia spp, a adição de conídios desse fungo em quantidades variáveis de 250 a 2500 conídios/g de fezes reduziu significativamente a quantidade das larvas (GRØNVOLD et al., 1985). Reduções de 86 a $96 \%$ no número de larvas infectantes de $\boldsymbol{C}$. oncophora foram observadas em bolos fecais e na vegetação circunvizinha após o tratamento com micélio de $A$. oligospora (GRØNVOLD et al., 1987). Reduções marcantes também foram observadas quando fezes contendo ovos de $\boldsymbol{O}$. ostertagi foram tratadas com o mesmo fungo (GRØNVOLD et al., 1988). Em um experimento subseqüente, GRØNVOLD et al. (1989) mostraram que bezerros que pastaram piquetes onde adicionaram-se bolos fecais inoculados com micélio de $\boldsymbol{A}$. oligospora, adquiriram $37 \%$ menos nematódeos e ganharam em média $16 \mathrm{~kg}$ a mais que os que pastaram piquetes onde os bolos fecais não foram inoculados.

A atividade predatória de outros isolados de $\boldsymbol{A}$. oligospora e fungos predadores de outras espécies do gênero Arthrobotrys, assim como espécies de outros gêneros, em cultivos, bolos fecais e pastagens foi demonstrada em vários experimentos (PANDEY, 1973; VIRAT \& PELOILLE, 1977; PELOILLE, 1981; NUNES et al., 1982; FERNANDEZ et al., 1985; HASHMI \& CONNAN, 1989; MENDOZA-DE GIVES et al., 1992, 1994; WALLER \& FAEDO, 1993; ARAÚJO et al., 1993; CHARLES et al., 1993; MENDOZA-DE GIVES \& VAZQUEZ-PRATZ, 1994). Mais recentemente ênfase tem sido dada a seleção de outras espécies e isolados de fungoṣ predadores. $\mathrm{Na}$ Austrália, bioensaios utilizando 94 espécies de fungos nematófagos identificaram como promissoras seis espécies do gênero Arthrobotrys, duas do gênero Geniculifera e duas do gênero Monacrosporum (WALLER \& FAEDO, 1993). No México, MENDOZA-DE GIVES \& VAZQUEZ-PRATZ (1994) verificaram que a adição de conídios de Monacrosporum eudermatum e $A$. oligospora em cultivos de fezes ovinas contendo ovos de $\boldsymbol{H}$. contortus promoveu reduções significativas no número de larvas infectantes. $O$ mesmo foi observado no Brasil, para A. oligospora e Arthrobotrys flagrans (CHARLES et al., 1993).
Seleção de fungos capazes de resistir a passagem pelo trato digestivo

A resistência à passagem pelo trato gastrintestinal é uma característica importante em fungos a serem usados no controle biológico, já que formulações para uso oral poderão ser desenvolvidas. SUPRONOV (1958) usando um isolado russo de $\boldsymbol{A}$. oligospora verificou que a cepa era capaz de crescer e exercer atividade predatória em larvas de nematódeos após a passagem pelo trato gastrintestinal de asininos. Resultados semelhantes foram observados por GRÜNER et al. (1985), PRYADKO \& OSIPOV (1986) e PELOILLE (1991) para Dactylaria candida, Candelabrella (Arthrobotrys) musiformis, Arthrobotrys tortor, Arthrobotrys arthrobotryoides e A. flagrans que sobreviveram à passagem pelo trato gastrintestinal de ovinos. Já um isolado de $\boldsymbol{A}$. oligospora e um de Dactylella bembicodes não sobreviveram à passagem pelo trato gastrintestinal de eqüinos e cobaias (DESCAZEAUX \& CAPELLE, 1939). Mais recentemente, GRØNVOLD et al. (1993) verificaram que o isolado sueco de $\boldsymbol{A}$. oligospora (ATCC24927), usado em uma série de experimentos na Dinamarca, não resistiu à passagem pelo trato gastrintestinal de bovinos, caprinos e suínos.

A existência de variação entre isolados de fungos nematófagos quanto a capacidade de sobreviver à passagem pelo trato gastrintestinal tem determinado o desenvolvimento de técnicas que permitam a seleção de fungos com esta característica. LARSEN et al. (1991) desenvolveram um bioensaio em que primeiramente os isolados são incubados em líquido ruminal diluído. As espécies que sobrevivem a esta seleção inicial são então testadas em saliva sintética, líquido ruminal simulando a atividade do rúmen, líquido ruminal após tratamento com pepsina e à solução de tripsina. Com o uso dessas técnicas LARSEN et al. (1991) selecionaram seis isolados de Arthrobotrys e sete de Duddingtonia. Esses isolados reduziram o desenvolvimento de larvas de $\boldsymbol{O}$. ostertagi em $75-96 \%$. Em seguida, LARSEN et al. (1992) submeteram esses isolados a testes in vivo através da administração oral diária dos fungos a bezerros. No quarto e quinto dia após o inicio da administração dos fungos, material fecal foi colhido das fezes desses bezerros e inoculado em placas de ágar-água. Em seguida, a atividade predatória dos fungos isolados do material fecal foi estudada através de bioensaios em bolos fecais e em cultivos de fezes. Dos fungos testados, dois do gênero Arthrobotrys e seis do gênero Duddingtonia reduziram o desenvolvimento de larvas de $\boldsymbol{O}$. ostertagi em 61 a 
93\% quando adicionados aos bolos fecais. A redução das larvas desse nematódeo nos cultivos fecais variou de 76 a $99 \%$.

$\mathrm{Na}$ Inglaterra, HASHMI \& CONNAN (1989) administraram oito milhões de conídios de um isolado local de $\boldsymbol{A}$. oligospora duas vezes por semana a bezerros infectados com $\boldsymbol{C}$. oncophora e $\boldsymbol{O}$. ostertagi e obtiveram reduçס̃es que variaram entre 51-62\% no número de larvas disponíveis nas pastagens utilizadas pelos bezerros tratados com $o$ fungo. Mais recentemente, WOLSTRUP et al. (1994), relataram um experimento onde uma cepa de Duddingtonia flagrans capaz de resistir à passagem pelo trato gastrintestinal foi administrada via oral a bezerros, diariamente, nos dois primeiros meses de pastoreio. Este grupo de bezerros e um outro grupo que não recebeu o fungo foram infectados experimentalmente com $\boldsymbol{O}$. ostertagi. A pastagem utilizada pelos bezerros que receberam o fungo tinha menos larvas infectantes disponíveis assim como os bezerros eliminavam menos ovos nas fezes. Ao final do período de observação eles pesavam em média $23 \mathrm{~kg}$ a mais que os que não receberam os fungos. A administração oral do mesmo isolado de fungo a bezerros mantidos em pastagens contaminadas por igual período, demonstrou que o fungo promoveu a diminuição da disponibilidade de larvas infectantes nas pastagens reduzindo a aquisição de Ostertagia sp. e Cooperia sp. no final da estação de pastoreio (LARSEN et al., 1995).

$\mathrm{Na}$ Austrália, WALLER et al. (1994), identificaram três espécies capazes de resistir à passagem pelo tubo gastrintestinal de ovinos: $\boldsymbol{A}$. oligospora, Arthrobotrys oviformis e Geniculifera eudermata. Conídios dessas espécies diluídos em água foram administrados a ovinos dotados de cânulas no abomaso e íleo. Os conídios foram capazes de passar pelos diferentes compartimentos do tubo gastrintestinal dos ovinos e exercer atividade predatória após a passagem.

Fungos nematófagos foram isolados em fezes frescas colhidas diretamente do reto ou em bolsas fecais. PARNELL \& GORDON (1963) relataram que o número de larvas infectantes em cultivos de fezes de ovinos infectados com $\boldsymbol{H}$. contortus declinou a níveis insignificantes devido a ação de Acrostalagmus sp. que eles isolaram das fezes frescas colhidas em bolsas fecais presas a esses ovinos. Mais tarde, a possibilidade de ocorrer naturalmente a passagem de fungo nematófago pelo trato gastrintestinal de ruminantes foi sugerida por HASHMI \& CONNAN (1989). LARSEN et al. (1993) em estudo desenvolvido na Austrália, efetuaram um levantamento que confirma a sugestão de HASHMI \& CONNAN (1989). No estudo, fezes de ruminantes eram colhidas do reto dos animais e submetidas a um bioensaio para identificar a presença de fungos nematófagos. Quarenta e oito isolados foram encontrados entre as 1742 amostras submetidas ao bioensaio. Dessas, apenas um isolado de D. flagrans foi capaz de resistir à passagem pelo trato gastrintestinal de ovinos em um teste in vivo e promover reduções significativas no número de larvas infectantes em cultivos de fezes de ovinos tratados via oral com este fungo. No Brasil, um levantamento semelhante encontra-se em andamento na EMBRAPA-Centro Nacional de Pesquisa de Gado de Leite. Até o momento, uma espécie foi identificada em fezes de um bovino colhidas diretamente do reto e uma em fezes colhidas em bolsas fecais fixadas em ovinos. A espécie isolada na fezes bovinas foi identificada como pertencente ao gênero Arthrobotrys enquanto a das fezes ovinas foi identificada como $A$. oligospora (SANTOS \& CHARLES, 1995b).

\section{CONCLUSÃO}

Há mais de um século sabe-se que alguns fungos exercem atividade predatória em nematódeos. No final da década de 30 os danos causados pelos nematódeos e a baixa eficiência dos métodos de controle da verminose existentes naquela época, impulsionaram alguns pesquisadores a iniciar estudos visando o controle biológico das formas de vida livre dos nematódeos com o uso de fungos nematófagos. Vários artigos foram publicados por pesquisadores franceses mostrando existir atividade de algumas espécies de fungos sobre larvas de nematódeos parasitas de animais (revisados por PANDEY, 1973). Eles também estudaram métodos de cultivo de fungos e recuperação e secagem de esporos com vistas à aplicação prática do controle biológico. ROUBAUD \& DESCHIENS (1941a; $1941 \mathrm{~b}$ ), mostraram atividade de fungos predadores sobre larvas de Strongyloides papillosus e Bunostomum sp. Mais de 20 anos se passaram desde a observação de ROUBAUD \& DESCHIENS até que PARNELL \& GORDON (1963) demonstraram o efeito de um fungo predador do gênero Acrostalagmus em larvas de Haemonchus contortus. Dez anos após, PANDEY (1973) publicou os resultados de uma série de experimentos demonstrando o efeito de dez fungos nematófagos contra larvas de Trichostrongylus axei e Ostertagia ostertagi.

Nos últimos cinco anos, as pesquisas sobre a utilização de fungos nematófagos se intensificaram. 
Os estudos iniciais de seleção utilizam metodologias simples. Técnicas in vitro sem sofisticação são disponíveis para selecionar isolados capazes de atrair nematódeos, produzir substâncias nematicidas, resistir à passagem pelo trato gastrintestinal e reduzir o número de larvas infectantes em cultivos, bolos fecais e vegetação. Fungos que demonstrarem resultados promissores nos testes preliminares são então submetidos a estudos adicionais para verificar a capacidade dos isolados em colonizar o bolo fecal em velocidade compatível com o desenvolvimento das larvas. Fungos capazes de colonizar, persistir e agir no habitat dos ovos e larvas, fáceis de serem produzidos em larga escala e armazenados, sem efeito indesejável ao serem associados aos produtos químicos comumente usados nas propriedades, seguro para o ser humano e sem efeito negativo no ambiente precisam ser selecionados. Muitos estudos deverão ser conduzidos após o estágio inicial de seleção de microrganismos tais como ensaios ecológicos e de manipulação microbiana. Estes estudos necessitarão de programas de pesquisa de longa duração e de um grande intercâmbio entre equipes e especialistas de diferentes áreas para que soluções práticas de emprego de microrganismos na higienização das pastagens possam ser obtidas.

Não se pretende com o controle biológico substituir o controle com o uso de fármacos. Os agentes de controle biológico raramente promovem a erradicação dos alvos. Geralmente eles reduzem a população a níveis aceitáveis. No controle das verminoses de ruminantes espera-se que o controle biológico possa ser empregado nas épocas em que as larvas infectantes são abundantes, reduzindo o número e conseqüentemente a incidência de casos clínicos, além de promover a imunidade dos animais através do desafio com um pequeno número de larvas.

\section{REFERÊNCIAS RIBLIOGRÁFICAS}

ARAÚJO, J.V., SANTOS, M. A., FERRAZ, S., et al. Antagonistic effect of predacious Arthrobotrys fungi on infective Haemonchus placei larvae. J Helminthol, v. 67, p. 136-138, 1993.

BARRON, G. L. The nematode-destroying fungi. Ontário: Canadian Biological Publications Ltd, 1977. 140p.

BJøRN, H. Workshop summary: anthelmintic resistance. Vet Parasitol, v. 54, n. 1-3, p. 321-325, 1994.

CHARLES, T.P., SANTOS, C. de P., ALVIM, G.P. Atividade predatória de duas espécies de fungos nematófagos nos estágios de vida livre dos nematódeos trichostrongilídeos Rev Bras Parasitol Vet, v. 2, n. 2, supl. 1, p. 46, 1993.
CHARLES, T.P., ROQUE, M. V. C., SANTOS, C. de P. Redução do número de larvas de Haemonchus contortus em fezes ovinas tratadas com conídios de Harposporium anguillulae. In: SEMINÁRIO DO COLÉGIO BRASILEIRO DE PARASITOLOGIA VETERINÁRIA, 9., 1995, Campo Grande. Campo Grande: CBPV, 1995.

DACKMAN, C., NORDBRING-HERTZ, B. Conidial traps - a new survival structure of nematode trapping fungus Arthrobotrys oligospora. Mycol Res, v. 96, n. 3, p. 194-198, 1992.

DESCAZEAUX, J., CAPELLE, C. Contribution à l'étude des champions prédateurs de larves de nématodes parasites des animaux domestiques. Bull I' Acad Vet France, v. 12, p. 284-288, 1939

DIJKSTERHUIS, J., HARDER, N., WYSS, U.; et al. Colonization and digestion of nematodes by the endoparasitic nematophagous fungus Drechmeria coniospora. Mycol Res, v. 95, n. 7, p. $873-878,1991$.

DONALD, A. D. Parasites, animal production and sustainable development. Vet Parasitol, v. 54, n. 1-3, p. 27-47, 1994.

FERNANDEZ, F. G., MEIRELES, M. C. A., COIMBRA, A. M Biological control of verminosis on ruminants. In: CONFERENCE OF THE WORLD ASSOCIATION FOR THE ADVANCEMENT OF VETERINARY PARASITOLOGY, 11., 1985. Rio de Janeiro. Abstracts... Rio de Janeiro: WAAVP, 1985. (Abstract 22).

GRøNVOLD, J., KORSHOLM, H., WOLSTRUP, J., et al. Laboratory experiments to evaluate the ability of Arthrobotrys oligospora to destroy infective larvae of Cooperia species, and to investigate the effect of physical factors on the growth of the fungus. J Helminthol, v. 59, p. $119-125,1985$.

GRØNVOLD, J., WOLSTRUP, J., HENRIKSEN, S.A., et al. Field experiments on the hability of Arthrobotrys oligospora (Hyphomycetales) to reduce the number of larvae of Cooperia oncophora (Trichostrongylidae) in cow pats and surrounding grass. J Helminthol, v. 61, p. 65-71, 1987

GRØNVOLD, J., NANSEN, P., HENRIKSEN, S.A., et al. The capacity of the predacious fungus Arthrobotrys oligospora (Hyphomycetales) to reduce numbers of infective larvae of Ostertagia ostertagi (Trichostrongylidae) in cow pats and herbage during the grazing season in Denmark. $J$ Helminthol, v. 62 , p. $271-280,1988$.

GRØNVOLD, J., HENRIKSEN, S.A., NANSEN, P., et al. Attempts to control infection with Ostertagia ostertagi (Trichostrongylidae) in grazing calves by adding mycelium of the nematode-trapping fungus Arthrobotrys oligospora (Hyphomycetales) to cow pats. J Helminthol, v. 63, p. 115126, 1989.

GRØNVOLD, J., WOLSTRUP, J., NANSEN, P., et al. Biological control of nematode parasites in cattle with nematodetrapping fungi: a survey of Danish studies. Vet Parasitol, v. 48, p. 311-325, 1993.

GRÜNER, L., PElOILle, M., SAUVÉ, C., et al. Survie et conservation de l'activité prédatrice vis-a-vis de nématodes trichostrongylides après ingestion par des Ovins de trois hyphomycétes prédateurs. C R Acad Sc, v. 300, n. 14, p. 525 $528,1985$. 
HASHMI, H.A., CONNAN, R.M. Biological control of ruminant trichostrongylid by Arthrobotrys oligospora, a predacious fungus. Parasitol Today, v. 5, n. 1., p. 28-30, 1989.

HERD, R., STRONG, L., WARDHAUGH, K. Environmental impact of avermectin usage in livestock. Vet Parasitol, v. 48, n. 1-4, p. 1-343, 1993.

JANSSON, H., JEYPRAKASH, A., ZUCKERMAN, B. M. Differential adhesion and infection of nematodes by the endoparasitic fungus Meria coniospora (Deuteromycetes). Appl Environ Microbiol, v. 49, n. 3, p. 552-555, 1985.

LARSEN, M., WOLSTRUP, J., HENRIKSEN, S.A., et al. In vitro stress selection of nematophagous fungi for biocontrol of parasitic nematodes in ruminants. J Helminthol, v. 65, p. 193-200, 1991

LARSEN, M., WOLSTRUP, J., HENRIKSEN, S.A., et al. In vivo passage through calves of nematophagous fungi selected for biocontrol of parasitic nematodes. J Helminthol, v. 66, p. 137-141, 1992

LARSEN, M., WALLER, P., FAEDO, M. The potential of nematophagous fungi to control the free living stages of nematode parasites of sheep: survey for the presence of fungi in fresh faeces of grazing livestock in Australia. Vet Parasitol, v. 53, p. 275-281, 1993

LARSEN, M., NANSEN, P., WOLSTRUP, J., et al. Biological control of trichostrongyles in calves by the fungus Duddingtonia flagrans fed to animals under natural grazing conditions. Vet Parasitol, 1995 (no prelo).

LÝSEK, H., KRAJŠí, D. Penetration of ovicidal fungus Verticilium chlamydosporium through the Ascaris lumbricoides egg-shells. Folia Parasitologica, v. 34, p. 5760,1987

MENDOZA-DE GIVES, P., VAZQUEZ-PRATS, V.M. Reduction of Haemonchus contortus infective larvae by three nematophagous fungi in sheep faecal cultures. Vet Parasitol, v. 55, p. $197-203,1994$.

MENDOZA-DE GIVES, P., ZAVALETA-MEJIA, E., HERRERA-RODRIGUEZ, D., et al. Interaction between the nematode destroying fungus Arthrobotrys robusta (Hyphomycetales) and Haemonchus contortus infective larvae in vitro. Vet Parasitol, v. 41, p. 101-107, 1992.

MENDOZA-DE GIVES, P., ZAVALETA-MEJIA, E., HERRERA-RODRIGUEZ, D., et al. In vitro trapping capability of Arthrobotrys spp. on infective larvae of Haemonchus contortus and Nocobbus aberrans. J Helminthol, v. 69, p. 223-229, 1994.

NANSEN, P., GRØNVOLD, J., HENRIKSEN, S.A., $\boldsymbol{e}$ t al. Predacious activity of the nematode-destroying fungus Arthrobotrys oligospora, on preparasitic larvae of Cooperia oncophora and on soil nematodes. Proc Helminthol Soc Washington, v. 53, n. 2, p. 237-243, 1986.

NANSEN, P., GRØNVOLD, J., HENRIKSEN, S.A., et al. Interactions between the predacious fungus Arthrobotrys oligospora and third-stage larvae of a series of animalparasitic nematodes. Vet Parasitol, v. 26, p. 329-337, 1988.
NORDBRING-HERTZ, B. Nematophagous fungi: strategies for nematode exploitation and survival. Microbiological Sciences, v. 5, n. 4, p. 108-116, 1988

NUNES, E.M., MEIRELES, M.C.A., FERNANDEZ, F.G Controle biologico: fungos e hyphomycetos no combate as formas pré-infestantes de nematódios. In: CONGRESSO BRASILEIRO DE MEDICINA VETERINÁRIA, 18., 1982, Camboriú. Resumos... Camboriú: Sociedade Brasileira de Medicina Veterinária, 1982. p. 150.

PANDEY, V.S. Predatory activity of nematode trapping fungi against the larvae of Trichostrongylus axei and Ostertagio ostertagi: a possible method of biological control. J Helminthol, v. 48, n. 1, p. 35-48, 1973.

PARNELL, I. W., GORDON, H.M.L. Predacious fungi: a possible method of biological control of parasitic nematodes. J Helminthol, v. 37, p. 339-342, 1963.

PELOILLE, M. Hyphomycètes prédateurs de nématodes dans une praire du Limousin. Entomophaga, v. 26, n. 1, p. 91-98, 1981.

PELOILLE, M. Selection of nematode-trapping fungi for use in biological control. In: KERRY, B.R., CRUMP, D.H. Integrated control of soil pests. Methods for studying nematophagous fungi. IOBC/WPRS Bulletin. XIV/2, 1991. p. 13-17

PRICHARD, R. Anthelmintic resistance. Vet Parasitol, v. 54, n. 1-3, p. 259-268, 1994.

PRYADKO, E. I., OSIPOV, P. P. Trials of nematophagous fungi in fields conditions. Biologicheskaya, v. 1, p. 30-33, 1986.

ROUBAUD, E., DESCHIENS, J. Action des Hyphomycetes prédateurs sur les larves de Synthétocaules et de Bunostomes. Bull Soc Path exot, v. 34, p. 127-130, 1941 a.

ROUBAUD, E., DESCHIENS, J. Essais relatifs à la prophylaxie de l'anguillulose du mouton par l'usage des Hyphomycètes prédateurs du sol. C R Acad Sc, v. 135, p. 687-690, 1941 b.

SANTOS, C. de P., CHARLES, T. P. Efeito da aplicação de conídios de Drechmeria coniospora em cultivos de fezes contendo ovos de Haemonchus contortus. Arq Bras Med Vet Zootec, v. 47, n. 2, p. 123-128, 1995a.

SANTOS, C. de P., CHARLES, T.P. Seleção de fungos nematófagos resistentes à passagem pelo trato digestivo de ruminantes. In: SEMINARIO DO COLÉGIO BRASILEIRO DE PARASITOLOGIA VETERINÁRIA, 9., 1995, Campo Grande. Campo Grande: CBPV, 1995b.

SOPRUNOV, F.F. Predacious hyphomycetes and their application in the control of pathogenic nematodes. Ashkhabad, 1958. 365p. Jerusalem: Israel Program for Scientific Translations, 1966, $292 \mathrm{pp}$. (traduzido do russo por NEMCHINOK, S.)

TUNLID, A., JANSSON, S. Proteases and their involvement in the infection and immobilization of nematodes by the nematophagous fungus Asthrobotrys oligospora. Appl Environ Microbiol, v. 57, n. 10, p. 2868-2872, 1991.

TUNLID, A., ROSEN, S., NORDBRING-HERTZ, B. Molecular mechanisms of adhesion in the nematophagous fungus Arthrobotrys oligospora. J Mycol Méd, v. 2, p. 36-42, 1992. 
VIRAT, M., PELOILLE, M. Pouvoir predateur in vitro d'une souche d'Arthrobotrys oligospora Fres. vis-a-vis d'un nematode zooparasitic. Ann Rech vét, v. 8, n. 1, p. 51-58, 1977.

WALLER, P.J. Prospects for biological control of nematode parasites of ruminants. NZ Vet J, v. 40, p. 1-3, 1992

WALLER, P. Workshop summary: Sustainable production systems. Vet Parasitol, v. 54, n. 1-3, p. 305-307, 1994.

WALLER, P.J., FAEDO, M. The potential of nematophagous fungi to control the free-living stages of nematode parasites of sheep: screening studies. Vet Parasitol, v. 49, p. 285-297, 1993.

WALLER, P.J., LARSEN, M., FAEDO, M. et al. The potential of nematophagous fungi to control the free-living stages of nematode parasites of sheep: in vitro and in vivo studies. Vet Parasitol, v. 51, p. 289-299, 1994.

WOLSTRUP, J., GRØNVOLD, J., HENRIKSEN, S.A., et al. An attempt to implement the nematode-trapping fungus Duddingtonia flagrans in biological control of trichostrongyle infections of first year grazing calves. $\mathbf{J}$ Helminthol, v. 68, p. 175-180, 1994.

Ciência Rural, v. 26, n. 2, 1996 